\title{
PROPAGATION OF $C^{\infty}$ REGULARITY FOR FULLY NONLINEAR SECOND ORDER STRICTLY HYPERBOLIC EQUATIONS IN TWO VARIABLES
}

\author{
BY
}

PAUL GODIN

\begin{abstract}
It is shown that if $u$ is a $C^{3}$ solution of a fully nonlinear second order strictly hyperbolic equation in two variables, then $u$ is $C^{\infty}$ at a point $m$ as soon as it is $C^{\infty}$ at some point of each of the two bicharacteristic curves through $m$. For semilinear equations, such a result was obtained before by Rauch and Reed if $u \in C^{1}$.
\end{abstract}

1. Introduction. In [2], Rauch and Reed proved the following result: if $u$ is a real $C^{1}$ solution of a strictly hyperbolic second order semilinear partial differential equation $\sum_{|\alpha|=2} a_{\alpha}(x) \partial^{\alpha} u+f(x, u, \nabla u)=0$ in an open subset $\Omega$ of $\mathbf{R}^{2}$ with $a_{\alpha}, f \in C^{\infty}$, then the $C^{\infty}$ singularities of $u$ in $\Omega$ propagate as in the linear case. More precisely if $m \in \Omega$ and if $\gamma_{1}$ and $\gamma_{2}$ are the two bicharacteristic curves of $\sum_{|\alpha|=2} a_{\alpha}(x) \partial^{\alpha}$ through $m$, the following holds: if $u \in C^{1}(\Omega, \mathbf{R})$ and $\gamma_{j} \backslash \operatorname{sing} \operatorname{supp} u \neq \varnothing$ for $j=1,2$, then $m \notin \operatorname{sing} \operatorname{supp} u$. In this note we shall show that Rauch and Reed's result still holds for completely nonlinear strictly hyperbolic partial differential equations of the second order in an open subset $\Omega$ of $\mathbf{R}^{2}$, provided that we assume that $u \in C^{3}(\Omega, \mathbf{R})$. This will be done by embedding $u$ and its derivatives up to order 2 in a solution of a suitable first order quasilinear system, following an idea of Lewy $[\mathbf{1}]$, and reducing then the problem to a semilinear one to which the results of $[2]$ can be applied.

2. Statement of the result. Let $\Omega$ be an open subset of $\mathbf{R}^{\mathbf{2}}$, with coordinates $(x, y)$, and let $u$ belong to $C^{3}(\Omega, \mathbf{R})$. Write $\nabla u=\left(\partial_{x} u, \partial_{y} u\right), \nabla^{2} u=$ $\left(\partial_{x}^{2} u, \partial_{x y}^{2} u, \partial_{y}^{2} u\right)$ and denote by $E$ a neighborhood in $\mathbf{R}^{8}$ of the set $\{(x, y, u(x, y)$, $\left.\left.\nabla u(x, y), \nabla^{2} u(x, y)\right),(x, y) \in \Omega\right\}$. Let $N=\left(N_{1}, N_{2}\right)$ belong to $\mathbf{R}^{2} \backslash\{0\}$ and assume that $F(x, y, z, p, q, r, s, t) \in C^{\infty}(E, \mathbf{R})$ is strictly hyperbolic in $\Omega$ at $u$, with respect to $N$; by this we mean that for all $(\xi, \eta) \in \mathbf{R}^{2} \backslash\{0\}$ not proportional to $N$ and all $(x, y) \in \Omega$, the roots $\lambda$ of the equation

$$
\tilde{F}_{r}(x, y)\left(\xi+\lambda N_{1}\right)^{2}+\tilde{F}_{s}(x, y)\left(\xi+\lambda N_{1}\right)\left(\eta+\lambda N_{2}\right)+\tilde{F}_{t}(x, y)\left(\eta+\lambda N_{2}\right)^{2}=0
$$

are real and distinct if $\tilde{F}_{r}(x, y)=\left(\partial_{r} F\right)\left(x, y, u(x, y), \nabla u(x, y), \nabla^{2} u(x, y)\right)$ and $\tilde{F}_{s}$, $\tilde{F}_{t}$ are defined similarly.

Recall that the null bicharacteristic strips of $\tilde{F}_{r} \partial_{x}^{2}+\tilde{F}_{s} \partial_{x y}^{2}+\tilde{F}_{t} \partial_{y}^{2}$ are the integral curves of the vector field $\partial_{\xi} f \partial_{x}+\partial_{\eta} f \partial_{y}-\partial_{x} f \partial_{\xi}-\partial_{y} f \partial_{\eta}$ on which $f=0$ if

Received by the editors October 4, 1984.

1980 Mathematics Subject Classification. Primary 35L70.

Key words and phrases. Propagation of regularity, second order fully nonlinear strictly hyperbolic equations in two variables. 
$f(x, y, \xi, \eta)=-\left(\tilde{F}_{r} \xi^{2}+\tilde{F}_{s} \xi \eta+\tilde{F}_{t} \eta^{2}\right)$. Since $u \in C^{3}$, it is easy to check that through each point $\left(x_{0}, y_{0}, \xi^{0}, \eta^{0}\right) \in f^{-1}(0)$ passes exactly one null bicharacteristic strip. The projection of the null bicharacteristic strips onto $(x, y)$ space are called bicharacteristic curves.

The purpose of this paper is to prove the following result.

THEOREM. Let $\Omega, F, u$ be as above. Assume that

$$
F\left(x, y, u(x, y), \nabla u(x, y), \nabla^{2} u(x, y)\right)=0 \quad \text { if }(x, y) \in \Omega .
$$

Denote by $\gamma_{1}, \gamma_{2}$ the two bicharacteristic curves of $\tilde{F}_{r} \partial_{x}^{2}+\tilde{F}_{s} \partial_{x y}^{2}+\tilde{F}_{t} \partial_{y}^{2}$ through $m \in \Omega$ and assume that for some $m_{1} \in \gamma_{1}, m_{2} \in \gamma_{2},\left\{m_{1}, m_{2}\right\} \cap \operatorname{sing} \operatorname{supp} u=\varnothing$. Then $m \notin \operatorname{sing} \operatorname{supp} u$.

3. Proof of the Theorem. After a linear change of variables, we may assume that $N=(1,0)$ and that $F$ is also strictly hyperbolic in $\Omega$ at $u$, with respect to $(0,1)$. An easy computation shows that the bicharacteristic curves of $\tilde{F}_{r} \partial_{x}^{2}+\tilde{F}_{s} \partial_{x y}^{2}+\tilde{F}_{t} \partial_{y}^{2}$ coincide, modulo a change of parametrization, with the integral curves of the vector fields $L_{j}=\partial_{x}+\rho_{j} \partial_{y}, j=1,2$, where $\rho_{j} \in C^{1}(\Omega, \mathbf{R})$ are the solutions of $\tilde{F}_{r} \rho^{2}-$ $\tilde{F}_{s} \rho+\tilde{F}_{t}=0$. The strict hyperbolicity assumption implies that $\rho_{1}(x, y) \neq \rho_{2}(x, y)$ for each $(x, y) \in \Omega$, so it is no restriction to assume that $\rho_{1}(x, y)<\rho_{2}(x, y)$ for each $(x, y) \in \Omega$. Denoting by $\varphi_{L_{j}}\left(t_{j}, m\right)$ the image of $m$ at time $t_{j}$ by the flow of $L_{j}$, we may assume that $m_{j}=\varphi_{L_{j}}\left(t_{j}, m\right)$ with $t_{j}>0, j=1,2$; the other cases are treated similarly. We put $\left[m, m_{j}\right]=\left\{\varphi_{L_{j}}(t, m), 0 \leq t \leq t_{j}\right\}, \gamma=\left[m, m_{1}\right] \cup\left[m, m_{2}\right]$.

To prove the announced theorem, it will be convenient to dispose of the following lemma, whose proof is given in the appendix at the end of the paper.

LEMMA 1. There exists a $C^{1}$ diffeomorphism $\Phi$ of an open neighborhood $W$ of $(0,0)$ in $\mathbf{R}^{2}$ onto an open neighborhood $V$ of $\gamma$ in $\Omega$, given by the equations $(x, y)=\Phi(\bar{x}, \bar{y})=(X(\bar{x}, \bar{y}), Y(\bar{x}, \bar{y}))$, such that $\Phi(0,0)=m$ and that the following holds if $\Psi=\Phi^{-1}$ :

(i) $\Phi \in C^{\infty}$ in an open neighborhood of $\Psi\left(m_{1}\right)=\left(\bar{x}_{0}, 0\right)$ and of $\Psi\left(m_{2}\right)=\left(0, \bar{y}_{0}\right)$;

(ii) $d \Psi\left(L_{1}\right)=f \partial_{\bar{x}}, d \Psi\left(L_{2}\right)=g \partial_{\bar{y}}$, where $f, g \in C(W)$;

(iii) $\partial_{\bar{y}} f, \partial_{\bar{x}} g \in C(W)$ and $f, g \in C^{\infty}$ in an open neighborhood of $\Psi\left(m_{1}\right)$ and $\Psi\left(m_{2}\right)$.

As announced in the introduction, we are now going to use an idea of Lewy [1] and embed $u$ and its derivatives up to order 2 in a solution of a quasilinear first order system. The system here will be obtained from $F$ and the diffeomorphism $\Phi$ of Lemma 1. Define the following functions of $x$ and $y$ :

$$
\left(\frac{d F}{d x}\right)=\tilde{F}_{x}+\tilde{F}_{z} \partial_{x} u+\tilde{F}_{p} \partial_{x}^{2} u+\tilde{F}_{q} \partial_{x y}^{2} u, \quad\left(\frac{d F}{d y}\right)=\tilde{F}_{y}+\tilde{F}_{z} \partial_{y} u+\tilde{F}_{p} \partial_{x y}^{2} u+\tilde{F}_{q} \partial_{y}^{2} u
$$

where $\tilde{F}_{x}(x, y)=\left(\partial_{x} F\right)\left(x, y, u(x, y), \nabla u(x, y), \nabla^{2} u(x, y)\right)$, and $\tilde{F}_{y}, \tilde{F}_{z}, \tilde{F}_{p}, \tilde{F}_{q}$ are defined analogously. Define now the following functions of $(\bar{x}, \bar{y}) \in W: U=u \circ \Phi, P=$ $\partial_{x} u \circ \Phi, Q=\partial_{y} u \circ \Phi, R=\partial_{x}^{2} u \circ \Phi, S=\partial_{x y}^{2} u \circ \Phi, T=\partial_{y}^{2} u \circ \Phi, \bar{\rho}_{j}=\rho_{j} \circ \Phi$, $j=1,2,\left(\mathcal{F}_{x}\right)=(d F / d x) \circ \Phi,\left(\mathcal{F}_{y}\right)=(d F / d y) \circ \Phi, \mathcal{F}_{r}=\tilde{F}_{r} \circ \Phi, \mathcal{F}_{s}=\tilde{F}_{s} \circ \Phi$. Then the following equations hold (see [1, p. 188]):

$$
\bar{\rho}_{1} \partial_{\bar{x}} X-\partial_{\bar{x}} Y=0
$$




$$
\begin{gathered}
P \partial_{\bar{x}} X+Q \partial_{\bar{x}} Y-\partial_{\bar{x}} U=0 \\
R \partial_{\bar{x}} X+S \partial_{\bar{x}} Y-\partial_{\bar{x}} P=0 \\
S \partial_{\bar{x}} X+T \partial_{\bar{x}} Y-\partial_{\bar{x}} Q=0 \\
\left(\mathcal{F}_{x}\right) \partial_{\bar{x}} X+\mathcal{F}_{r} \partial_{\bar{x}} R+\left(\mathcal{F}_{s}-\mathcal{F}_{r} \bar{\rho}_{1}\right) \partial_{\bar{x}} S=0 \\
\left(\mathcal{F}_{y}\right) \partial_{\bar{x}} X+\mathcal{F}_{r} \partial_{\bar{x}} S+\left(\mathcal{F}_{s}-\mathcal{F}_{r} \bar{\rho}_{1}\right) \partial_{\bar{x}} T=0 \\
\bar{\rho}_{2} \partial_{\bar{y}} X-\partial_{\bar{y}} Y=0 \\
\left(\mathcal{F}_{x}\right) \partial_{\bar{y}} X+\mathcal{F}_{r} \partial_{\bar{y}} R+\left(\mathcal{F}_{s}-\mathcal{F}_{r} \bar{\rho}_{2}\right) \partial_{\bar{y}} S=0 .
\end{gathered}
$$

Equalities (1)-(8) are proved in [1] (where a different coordinate system is used; here we have to work with coordinates adapted to the study of the propagation of singularities). All formulas except (5), (6) and (8) are immediate consequences of the change of variables $\Phi$. Let us repeat briefly the argument of [1] which leads to (5). Differentiating the equation $F\left(x, y, u(x, y), \nabla u(x, y), \nabla^{2} u(x, y)\right)=0$ with respect to $x$, we obtain

$$
\left(\frac{d F}{d x}\right)+\tilde{F}_{r} \partial_{x}^{3} u+\tilde{F}_{s} \partial_{x}^{2} \partial_{y} u+\tilde{F}_{t} \partial_{x} \partial_{y}^{2} u=0
$$

Since $\tilde{F}_{r} \rho_{1}^{2}-\tilde{F}_{s} \rho_{1}+\tilde{F}_{t}=0,(9)$ implies that

$$
\left(\frac{d F}{d x}\right)+\tilde{F}_{r} L_{1} \partial_{x}^{2} u+\left(\tilde{F}_{s}-\tilde{F}_{r} \rho_{1}\right) L_{1} \partial_{x y}^{2} u=0
$$

which gives (5). The proofs of (6) and (8) are similar (see [1]). Writing $v=$ $\left(v_{1}, v_{2}, v_{3}, v_{4}, v_{5}, v_{6}, v_{7}, v_{8}\right)=(X, Y, U, P, Q, R, S, T)$, we see that $v$ is a solution of a system of the type

$$
\begin{array}{ll}
\sum_{1 \leq j \leq 8} a_{i j}(v) \partial_{\bar{x}} v_{j}=0, & 1 \leq i \leq 6 \\
\sum_{1 \leq j \leq 8} a_{i j}(v) \partial_{\bar{y}} v_{j}=0, & i=7,8
\end{array}
$$

where $\operatorname{det}\left(a_{i j}\right)_{1 \leq i, j \leq 8}=\mathcal{F}_{r}^{2}\left(\mathcal{F}_{s}-\mathcal{F}_{r} \bar{\rho}_{1}\right)\left(\bar{\rho}_{2}-\bar{\rho}_{1}\right)^{2}$ never vanishes in $W$ (because the strict hyperbolicity of $F$ at $u$ with respect to $(1,0)$ and $(0,1)$ implies that $\tilde{F}_{r}$ and $\tilde{F}_{t}=\rho_{1}\left(\tilde{F}_{s}-\tilde{F}_{r} \rho_{1}\right)$ never vanish in $\left.\Omega\right)$. Notice that $a_{i j} \in C^{\infty}$ in a neighborhood of the range of $v$ since $F \in C^{\infty}$ and $\rho_{1}, \rho_{2}$ are the solutions of $\tilde{F}_{r} \rho^{2}-\tilde{F}_{s} \rho+\tilde{F}_{t}=0$. Since $u \in C^{\infty}$ in a neighborhood of $m_{1}$ and $m_{2}$, Lemma 1(i) shows that $v \in C^{\infty}$ in a neighborhood of $\left(\bar{x}_{0}, 0\right)$ and $\left(0, \bar{y}_{0}\right)$. We are going to show the following:

$$
v \in C^{\infty} \text { in a neighborhood of }(0,0) \text {. }
$$

From (12) it will follow in particular that $X, Y, U \in C^{\infty}$ in a neighborhood of $(0,0)$. This of course will imply that $u \in C^{\infty}$ in a neighborhood of $m$, completing the proof of the Theorem. Now (12) is a consequence of the following lemma, where we write $x, y$ instead of $\bar{x}, \bar{y}$ for simplicity.

LEMMA 2. Put $A=((-\varepsilon, \varepsilon) \times(-M, \varepsilon)) \cup((-M, \varepsilon) \times(-\varepsilon, \varepsilon)) \subset \mathbf{R}^{2}$, where $\varepsilon, M>0$. Let $v=\left(v_{1}, \ldots, v_{m}\right) \in C^{1}\left(A, \mathbf{R}^{m}\right)$ be a solution of the quasilinear system

$$
\sum_{1 \leq j \leq m} a_{i j}(x, y, v) \partial_{x} v_{j}=b_{i}(x, y, v), \quad 1 \leq i \leq r
$$




$$
\sum_{1 \leq j \leq m} a_{i j}(x, y, v) \partial_{y} v_{j}=b_{i}(x, y, v), \quad r+1 \leq i \leq m,
$$

where $a_{i j}, b_{i} \in C^{\infty}(A \times B)$; here $B$ is an open reighborhood of $v(A)$ in $\mathbf{R}^{m}$ and $\operatorname{det}\left(a_{i j}\right)_{1 \leq i, j \leq m}$ never vanishes in $A \times B$. Then, if $v \in C^{\infty}$ in $((-\varepsilon, \varepsilon) \times(-M,-M+$ $\varepsilon)) \cup((-M,-M+\varepsilon) \times(-\varepsilon, \varepsilon))$, it follows that $v \in C^{\infty}$ in $(-\varepsilon, \varepsilon) \times(-\varepsilon, \varepsilon)$.

ProOF OF Lemma 2. Denote by $D_{x}, D_{y}$ the distribution derivatives with respect to $x$ and $y$ respectively. Note that if $f \in C^{1}$ and $g \in C$, one has $D_{x}(f g)=$ $g D_{x} f+f D_{x} g$, where $f D_{x} g$ should be interpreted as the product of a $C^{1}$ function and a distribution of order $\leq 1$, which makes sense. Differentiating in the distribution sense (13) with respect to $y$ and (14) with respect to $x$, we obtain

$$
\begin{aligned}
\sum_{1 \leq j \leq m} a_{i j}(x, y, v) D_{y} \partial_{x} v_{j}= & -\sum_{1 \leq j \leq m} D_{y}\left(a_{i j}(x, y, v)\right) \partial_{x} v_{j} \\
& +D_{y}\left(b_{i}(x, y, v)\right), \quad 1 \leq i \leq r, \\
\sum_{1 \leq j \leq m} a_{i j}(x, y, v) D_{x} \partial_{y} v_{j}=- & \sum_{1 \leq j \leq m} D_{x}\left(a_{i j}(x, y, v)\right) \partial_{y} v_{j} \\
& +D_{x}\left(b_{i}(x, y, v)\right), \quad r+1 \leq i \leq m,
\end{aligned}
$$

where the right-hand sides are continuous functions of $(x, y)$ and the distribution derivatives of the composite $C^{1}$ functions $a_{i j}(x, y, v)$ and $b_{i}(x, y, v)$ coincide with the usual derivatives and can be computed by the chain rule. Since $\operatorname{det}\left(a_{i j}\right)_{1 \leq i, j \leq m}$ does not vanish, (15) and (16) imply that

$$
D_{x y}^{2} v_{j}=A_{j}(x, y, v, \nabla v), \quad 1 \leq j \leq m,
$$

where $A_{j} \in C^{\infty}\left(A \times B \times \mathbf{R}^{2 m}\right)$. Now the proof of Theorem 3 of [2] shows that for all $k \in \mathbf{Z}^{+}, D_{x}^{k} v$ and $D_{y}^{k} v \in C((-\varepsilon, \varepsilon) \times(-\varepsilon, \varepsilon))$. Hence $v \in C^{\infty}((-\varepsilon, \varepsilon) \times(-\varepsilon, \varepsilon))$. This proves Lemma 2 and completes the proof of the Theorem.

Appendix: Proof of Lemma 1. First we prove the following result, which will be useful in the proof of Lemma 1.

LEMMA A.1. One can find a neighborhood $Z$ of $\gamma$ in $\Omega$ and two strictly positive functions $\theta_{1}, \theta_{2} \in C(Z, \mathbf{R})$ such that the following holds:

(i) $L_{2} \theta_{1}$ and $L_{1} \theta_{2}$ exist and belong to $C(Z)$.

(ii) $\left[\theta_{1} L_{1}, \theta_{2} L_{2}\right]=0$ in $Z$.

(iii) Both $\theta_{1}$ and $\theta_{2}$ are $C^{\infty}$ in a neighborhood of $\left\{m_{1}, m_{2}\right\}$.

PROOF OF LEMMA A.1. It is no restriction to assume that $m=(0,0)$. We can obtain $\theta_{1}$ and $\theta_{2}$ as solutions of Cauchy problems with initial data on a suitable noncharacteristic curve $\Gamma$ linking $m_{1}$ and $m_{2}$. For the construction of $\Gamma$ and also for other purposes later in this appendix it is convenient to make a suitable change of coordinates. So let $\tilde{\rho}_{1} \in C_{0}^{1}\left(\mathbf{R}^{2}, \mathbf{R}\right)$ be equal to $\rho_{1}$ in an open neighborhood $U_{1}$ of $\gamma$ and define the $C^{1}\left(\mathbf{R}^{2}, \mathbf{R}^{2}\right)$ diffeomorphism $\chi_{1}(x, y)=(x, \tilde{y}(x, y))$ by the equations $\partial_{x} y=\tilde{\rho}_{1}(x, y), y=\tilde{y}$ if $x=0$. A simple computation shows that, in $\chi_{1}\left(U_{1}\right), d \chi_{1}\left(L_{1}\right)=\partial_{x}, d \chi_{1}\left(L_{2}\right)=\partial_{x}+B \partial_{\tilde{y}}\left(\right.$ where $B$ and $\partial_{x} B \in C\left(\chi_{1}(\Omega)\right)$ ), and $B>0$. Choose a real valued function $B^{\prime}(x, \tilde{y})$ with the following properties: $B^{\prime}$ and $\partial_{x} B^{\prime} \in C\left(\mathbf{R}^{2}\right), B^{\prime}$ is equal to $B$ in an open neighborhood $U_{2}$ of $\chi_{1}(\gamma)$ 
contained in $\chi_{1}\left(U_{1}\right), B^{\prime}$ never vanishes and is constant outside a compact subset of $\chi_{1}(\Omega)$. Define the $C^{1}\left(\mathbf{R}^{2}, \mathbf{R}^{2}\right)$ diffeomorphism $\chi_{2}(x, \tilde{y})=(\tilde{x}(x, \tilde{y}), \tilde{y})$ by the equations $\partial_{\tilde{y}} x=1 / B^{\prime}(x, \tilde{y}), x=\tilde{x}$ if $\tilde{y}=0$. Write $\chi=\chi_{2} \circ \chi_{1}$ and put $\tilde{G}=\chi_{2}\left(U_{2}\right)$. Then $d \chi\left(L_{1}\right)=a \partial_{\tilde{x}}$ and $d \chi\left(L_{2}\right)=b \partial_{\tilde{y}}$ in $\tilde{G}$, where $a, b>0$ in $\tilde{G}$, and $a, \partial_{\tilde{y}} a, b, \partial_{\tilde{x}} b \in$ $C(\tilde{G})$. We have $\chi\left(m_{1}\right)=(\alpha, 0), \chi\left(m_{2}\right)=(0, \beta)$ for some $\alpha, \beta \in \mathbf{R} \backslash\{0\}$. Since $m_{j}=\varphi_{L_{j}}\left(t_{j}, m\right)$ with $t_{j}>0$ and since $a, b>0$, it follows that $\alpha>0, \beta>0$. Choose $\delta>0$ so small that the set $\tilde{G}^{\prime}=\left\{(\tilde{x}, \tilde{y}) \in \mathbf{R}^{2},|\tilde{y}|<\delta,-\delta<\tilde{x}<\alpha+\delta\right\} \cup\{(\tilde{x}, \tilde{y}) \in$ $\left.\mathbf{R}^{2},|\tilde{x}|<\delta,-\delta<\tilde{y}<\beta+\delta\right\}$ is contained in $\tilde{G}$. For $j=1,2$, let $\omega_{j}$ be an arc of noncharacteristic curve having $m_{j}$ as an interior point, $C^{\infty}$ in the coordinate system $x, y$, such that $\chi\left(\omega_{j}\right)$ is given by the equation $\tilde{y}=f_{j}(\tilde{x})$ for $\left|\tilde{x}-\tilde{x}\left(\chi\left(m_{j}\right)\right)\right|<\delta^{\prime}$, with $0<\delta^{\prime} \leq \delta$ and $d f_{j} / d \tilde{x}<0$. Define $f \in C^{1}\left(\left[-\delta^{\prime} / 2, \alpha+\delta^{\prime} / 2\right]\right)$ such that $f=f_{2}$ close to $0, f=f_{1}$ close to $\alpha, d f / d \tilde{x}<0$, and the graph $\tilde{\Gamma}$ of $f$ is contained in $\tilde{G}^{\prime}$. Write $\Gamma=\chi^{-1}(\tilde{\Gamma})$.

Using $\Gamma$ we may construct $\theta_{1}$ and $\theta_{2}$ with the required properties. Let $V_{j} \subset \Omega$, $j=1,2$, be open neighborhoods of $m_{j}$ such that sing supp $u \cap V_{j}=\varnothing$ and $\Gamma \in$ $C^{\infty}$ in $V_{j}$. Since $L_{1}$ and $L_{2}$ are linearly independent in $\Omega$, we have $\left[L_{1}, L_{2}\right]=$ $\lambda_{1} L_{1}+\lambda_{2} L_{2}$ in $\Omega$, with $\lambda_{1}, \lambda_{2} \in C(\Omega)$; actually $\lambda_{1}, \lambda_{2} \in C^{\infty}\left(V_{1}\right) \cap C^{\infty}\left(V_{2}\right)$ since sing supp $u \cap V_{j}=\varnothing, j=1,2$. Put $\tilde{Z}=\left\{(\tilde{x}, \tilde{y}) \in \tilde{G}^{\prime},-\delta^{\prime} / 2<\tilde{x}<\alpha+\delta^{\prime} / 2\right.$, $\left.f\left(\alpha+\delta^{\prime} / 2\right)<\tilde{y}<f\left(-\delta^{\prime} / 2\right)\right\}, Z=\chi^{-1}(\tilde{Z})$, and define $\beta_{1}, \beta_{2}$ in $Z$ by the Cauchy problems $L_{2} \beta_{1}=\lambda_{1}$ in $Z, \beta_{1}=0$ on $\Gamma, L_{1} \beta_{2}=-\lambda_{2}$ in $Z, \beta_{2}=0$ on $\Gamma$. For $j=1,2$, put $\theta_{j}=e^{\beta_{j}}, M_{j}=\theta_{j} L_{j}$. Then $\theta_{j} \in C^{\infty}$ in a neighborhood of both $m_{1}$ and $m_{2}$, and $\left[M_{1}, M_{2}\right]=0$ in $Z$. The proof of Lemma A.1 is complete.

REMARK. For the sequel, it is useful to remark that the following holds:

$$
\begin{aligned}
& d \chi\left(M_{1}\right)=J_{1} \partial_{\tilde{x}} \text { and } d \chi\left(M_{2}\right)=J_{2} \partial_{\tilde{y}} \text { in } \tilde{Z}, \text { where } J_{1}, J_{2} \in C(\tilde{Z}), \\
& J_{1}>0 \text { is independent of } \tilde{y} \text { and } J_{2}>0 \text { is independent of } \tilde{x} .
\end{aligned}
$$

In fact, since $d \chi\left(M_{1}\right)=\left(\theta_{1} \circ \chi^{-1}\right) a \partial_{\tilde{x}}$ and $d \chi\left(M_{2}\right)=\left(\theta_{2} \circ \chi^{-1}\right) b \partial_{\tilde{y}}$ in $\tilde{Z}$ with $a$ and $b$ as in the proof of Lemma A.1, $\left[d \chi\left(M_{1}\right), d \chi\left(M_{2}\right)\right]$ makes sense as well as $\left[d \chi\left(L_{1}\right), d \chi\left(L_{2}\right)\right]$. To prove $(18)$ it suffices to show that $\left[d \chi\left(M_{1}\right), d \chi\left(M_{2}\right)\right]=$ 0 in $\tilde{Z}$, which is a consequence of the definition of $\lambda_{1}, \lambda_{2}$ and of the formula $\left[d \chi\left(L_{1}\right), d \chi\left(L_{2}\right)\right]=\sum_{1 \leq j \leq 2}\left(\lambda_{j} \circ \chi^{-1}\right) d \chi\left(L_{j}\right)$ in $\tilde{Z}$. The latter equality follows immediately from the fact that

$$
\left[d \chi\left(L_{1}\right), d \chi\left(L_{2}\right)\right]=d \chi\left(\left[L_{1}, L_{2}\right]\right) \text { in } \tilde{Z} .
$$

If $\chi$ were of class $C^{2},(19)$ would of course be trivial, but here $\chi$ is only of class $C^{1}$. However an explicit computation of both sides of (19), using the precise definition of $\chi$, shows that (19) holds. Hence (18) holds.

We can now prove Lemma 1 . Since $\theta_{1}, \theta_{2}>0$ in $Z$, we have $\varphi_{M_{1}}\left(\bar{x}_{0}, m\right)=m_{1}$, $\varphi_{M_{2}}\left(\bar{y}_{0}, m\right)=m_{2}$ for some unique $\bar{x}_{0}>0, \bar{y}_{0}>0$, where as before $\varphi_{M}(t, p)$ means the image of $p$ at time $t$ by the flow of the vector field $M . \varphi_{M_{j}}, j=1,2$, is well defined since $\varphi_{d \chi\left(M_{j}\right)}$ is well defined. For $\varepsilon>0$ put $W_{\varepsilon}=\left\{(\bar{x}, \bar{y}) \in \mathbf{R}^{2},-\varepsilon<\bar{x}<\right.$ $\left.\bar{x}_{0}+\varepsilon,|y|<\varepsilon\right\} \cup\left\{(\bar{x}, \bar{y}) \in \mathbf{R}^{2},|\bar{x}|<\varepsilon,-\varepsilon<\bar{y}<\bar{y}_{0}+\varepsilon\right\}$. (18) shows that if $\varepsilon>0$ is small enough, the functions

$$
(\bar{x}, \bar{y}) \mapsto \varphi_{d \chi\left(M_{1}\right)}\left(\bar{x}, \varphi_{d \chi\left(M_{2}\right)}(\bar{y}, \chi(m))\right)
$$

and

$$
(\bar{x}, \bar{y}) \mapsto \varphi_{d \chi\left(M_{2}\right)}\left(\bar{y}, \varphi_{d \chi\left(M_{1}\right)}(\bar{x}, \chi(m))\right)
$$


are $C^{1}$ of $W_{\varepsilon}$ into $\tilde{Z}$ and are equal. Therefore if we put

$$
\Phi(\bar{x}, \bar{y})=\varphi_{M_{1}}\left(\bar{x}, \varphi_{M_{2}}(\bar{y}, m)\right),
$$

both sides of equality (20) below are well defined and we have

$$
\Phi(\bar{x}, \bar{y})=\varphi_{M_{2}}\left(\bar{y}, \varphi_{M_{1}}(\bar{x}, m)\right)
$$

if $(\bar{x}, \bar{y}) \in W_{\varepsilon}$. The definition of $\Phi$ implies that

$$
\partial_{\bar{x}} \Phi(\bar{x}, \bar{y})=M_{1}(\Phi(\bar{x}, \bar{y})) \quad \text { if }(\bar{x}, \bar{y}) \in W_{\varepsilon},
$$

and (20) shows that

$$
\partial_{\bar{y}} \Phi(\bar{x}, \bar{y})=M_{2}(\Phi(\bar{x}, \bar{y})) \quad \text { if }(\bar{x}, \bar{y}) \in W_{\varepsilon} .
$$

(21) and (22) show that $\Phi$ has rank 2 at each point of $W_{\varepsilon}$. The function $(\bar{x}, \bar{y}) \mapsto$ $\varphi_{d \chi\left(M_{1}\right)}\left(\bar{x}, \varphi_{d \chi\left(M_{2}\right)}(\bar{y}, \chi(m))\right)$ is clearly injective in $W_{\varepsilon}$, and so is $\Phi$. Hence $\Phi$ is a $C^{1}$ diffeomorphism of $W_{s}$ onto an open neighborhood of $\gamma$. Since for $j=1,2$, $M_{j} \in C^{\infty}$ close to $m_{1}$ and close to $m_{2}$, it follows from (21), (22) that $\Phi \in C^{\infty}$ close to $\left(\bar{x}_{0}, 0\right)$ and $\left(0, \bar{y}_{0}\right)$, which proves Lemma $1(\mathrm{i})$. (21) and (22) also immediately imply (ii), with

$$
f=1 /\left(\theta_{1} \circ \Phi\right), \quad g=1 /\left(\theta_{2} \circ \Phi\right) .
$$

(iii) follows readily from (23). The proof of Lemma 1 is complete.

\section{REFERENCES}

1. H. Lewy, Über das Anfangswertproblem einer hyperbolischer nichtlinearen partiellen Differentialgleichung zweiter Ordnung mit zwei unabhängigen Veränderlichen, Math. Ann. 98 (1927), 179-191.

2. J. Rauch and M. C. Reed, Propagation of singularities for semilinear hyperbolic equations in one space variable, Ann. of Math. (2) 111 (1980), 531-552.

Département de MATHÉmatique, Université libre de BRUXelles, Campus De la Plaine C.P. 214, Boulevard du Triomphe, 1050 Bruxelles, Belgium 